\title{
MODELLING OF THE CARDIOPULMONARY RESPONSES TO MAXIMAL AEROBIC EXERCISE IN PATIENTS WITH CYSTIC FIBROSIS
}

1 Craig A. Williams ${ }^{1 *}$, Kyle C. A. Wedgwood ${ }^{2,3}$, Hossein Mohammadi ${ }^{2,4}$, Owen W. Tomlinson ${ }^{1}$, 2 Krasimira Tsaneva-Atanasova ${ }^{2,3}$

$4{ }^{1}$ Children's Health and Exercise Research Centre, Sport and Health Sciences, University of Exeter, 5 Exeter, UK

$6 \quad{ }^{2}$ Department of Mathematics and Living Systems Institute, University of Exeter, Exeter, UK

$7 \quad{ }^{3}$ Centre for Biomedical Modelling and Analysis, University of Exeter, Exeter, EX4 4QJ

$8{ }^{4}$ EPSRC Centre for Predictive Modelling in Healthcare, University of Exeter, Exeter, EX4 4QJ, UK

10 * Corresponding Author:

11 Professor Craig A. Williams

12 Children's Health and Exercise Research Centre

13 Sport and Health Sciences

14 College of Life and Environmental Sciences

15 University of Exeter

16 St Luke's Campus

17 Exeter, EX1 2LU

18 UNITED KINGDOM

19 Tel: 44 (0)1392 724890

20 Email: c.a.williams@exeter.ac.uk

22 Keywords: modelling, Gaussian process, ventilation, gas exchange, physiology 
ABSTRACT

Cystic fibrosis (CF) is a debilitating chronic condition, which requires complex and expensive disease management. Exercise has now been recognised as a critical factor in improving health and quality of life in patients with CF. Hence, cardiopulmonary exercise testing (CPET) is used to determine aerobic fitness of young patients as part of the clinical management of CF. However, at present there is a lack of conclusive evidence for one limiting system of aerobic fitness for CF patients at individual patient level.

Here, we perform detailed data analysis that allows us to identify important systems-level factors that affect aerobic fitness. We use patients' data and principal component analysis to confirm the dependence of CPET performance on variables associated with ventilation and metabolic rates of oxygen consumption. We find that the time at which participants cross the anaerobic threshold (AT) is well correlated with their overall performance. Furthermore, we propose a predictive modelling framework that captures the relationship between ventilatory dynamics, lung capacity and function and performance in CPET within a group of children and adolescents with CF. Specifically, we show that using Gaussian processes (GP) we can predict AT at the individual patient level with reasonable accuracy given the small sample size of the available group of patients. We conclude by presenting future perspectives for improving and extending the proposed framework.

Our modelling and analysis have the potential to pave the way to designing personalised exercise programmes that are tailored to specific individual needs relative to patient's treatment therapies. 


\section{INTRODUCTION}

63 Cystic fibrosis (CF) is the most common life shortening genetic disease in the Caucasian population, 64 affecting nearly 11,000 individuals in the United Kingdom (UK) (1). The pathology of the disease, for which there is no cure, manifests itself throughout the respiratory, digestive and reproductive systems of the human body. Currently, as there is no cure for CF, the management of the disease is a key factor in the quality of care and health related quality of life factors. Part of the management of this disease requires cardiopulmonary exercise testing (CPET) to determine aerobic fitness, as represented by the maximal oxygen consumption $\left(\dot{\mathrm{VO}}_{2 \mathrm{max}}\right)$. This parameter provides a clinically useful prognostic evaluation of a patient's functional capabilities. Even in mild to moderate severity of CF, patients are known to demonstrate impairments in cardiac and respiratory functions leading to exercise intolerance.

Enhanced aerobic fitness has been shown to improve quality of life in young patients with CF (6-18 years), with benefits including lower risk of hospitalisation, increased exercise tolerance, reduced residual volume, increased endurance of the respiratory muscles, enhanced sputum expectoration and decreased rate of decline in pulmonary function (2-6). It has also been shown from CPET that individuals with $\mathrm{CF}$ possessing a higher $\dot{\mathrm{V}}_{2 \max }$ are shown to have a reduced mortality risk. Nixon et al. (1992) reported that individuals with a $\dot{\mathrm{V}}{ }_{2 \max }$ greater than $82 \%$ of their predicted value had an $83 \%$ 8-year survival rate, compared to just 28\% 8-year survival rate for patients with a $\mathrm{V}_{2 \max }$ less than $58 \%$ of their predicted value (7). Furthermore, patients with a higher $\dot{\mathrm{V}} \mathrm{O}_{2 \max }$ also have additional benefits in terms of improved fluid balance, retention of serum electrolytes through increased plasma volume, and potential for impact on sweat gland function thus reducing thermal strain and dehydration (8). These systemic changes are advantageous responses to exercise training, and result in an enhanced quality of life, increased physical function and increased life expectancy $(9$, $10)$.

Effective management of the disease has become even more critical in recent years due to an aging CF population group, with the median predicted survival of children born with CF in the UK now being 45 years (1). As a consequence of an aging patient group and high medical care costs (11), maintaining or enhancing fitness is crucial. Exercise has being widely acknowledged as a key management strategy for $\mathrm{CF}$, supported by some mechanistic data on the systemic effects of exercise at the cellular level in vivo in young patients with CF (5-9). However, an integrated systems level 
understanding of the limitations of aerobic fitness for CF patients is lacking. Measurement techniques that do exist to quantify within-organ, real-time perfusion and intracellular oxygenation are invasive and unethical for use with paediatric patients, and current animal model research provides limited direct relevance to paediatric pathology. In clinical practice, there is significant interaction between cardiac and pulmonary function and the behaviour of the systemic vasculature during exercise training. This can result in the functional improvement in one part of the combined system, but detrimental effects on others (9). Clinicians therefore inevitably have to adopt very imprecise guidelines related to exercise prescription (12).

The use of modelling and simulation tools in clinical medicine is currently the subject of intense research interest both in the UK and internationally (13-17), and the adoption of a systems biomedicine approach to build and validate novel multi-scale, organ-level, integrated, re-usable and re-deployable models represents a paradigm shift in biomedical modelling and simulation. There are numerous organ level models in existence (18-21), however, to date there have been limited attempts to either integrate these or to apply them to real clinical applications. There is ongoing basic science and clinical trial work providing data on the micro (22) and macrovascular (23) changes associated with exercise. These data, although important, have yet to be integrated quantitatively with other data streams. In particular, there has been almost no previous work on the use of predictive modelling and simulation technologies for developing treatment strategies for CF patients.

114 Here, we present detailed data analysis of responses to progressive exercise in patients with CF, with

115 a view of determining predictors of performance. We find that the time at which participants cross

116 the anaerobic threshold (AT), as measured by means of gas exchange threshold (GET) is well 117 correlated with overall performance. To gain further insight, we then develop a surrogate (statistical)

118 model that allows us to evaluate how CF impairs exercise tolerance relative to increasing ventilatory 119 and metabolic demands. Our modelling and analysis was based on data collected during cycling 120 exercise form a CPET at different work rates (from resting to voluntary exhaustion) in young patients 121 with CF. The outputs produced are discussed in this paper, with analyses focussing on pulmonary parameters.

\section{METHODS}

125 Before turning our attention to modelling, we perform an exploratory analysis of the available data in 126 order to identify predictors of performance. This study was carried out in accordance with the 
recommendations of the European Respiratory Society, written consent and assent was obtained from

128 parent(s)/guardian(s) and participants, respectively. All participants' parents gave written informed

129 consent in accordance with the Declaration of Helsinki. The protocol was approved by the South

130 West NHS Research Ethics Committee. Data were collected from 15 children and adolescents with

$131 \mathrm{CF}$, who performed a valid (24) and reliable (25) combined ramp incremental and supramaximal

132 (Smax) CPET to determine $\dot{\mathrm{V}} \mathrm{O}_{2 \max }$ and the GET. This protocol was performed on an electronically

133 braked cycle ergometer, and required patients to perform an initial exhaustive ramp incremental test

134 at a pre-determined rate between $10-25 \mathrm{~W} \cdot \mathrm{min}^{-1}$, in order to elicit exhaustion in approximately ten

135 minutes (26). After a 3-min warm-up at 10-20 W, participants completed this incremental test to the

136 point of volitional exhaustion, maintaining a cadence of 70-80 rpm throughout. Exhaustion was

137 defined as a $10 \mathrm{rpm}$ drop in cadence for five consecutive seconds, despite strong verbal

138 encouragement. Active (5-min cycling at $20 \mathrm{~W})$ and then passive seated recovery (10 min) then

139 preceded the Smax bout. Smax verification consisted of a 3-min warm-up (10-20 W), followed by a

140 "step" transition to a constant work rate corresponding to $110 \%$ peak power output (27). Upon

141 volitional exhaustion (defined previously), a 5-min active recovery (slow cycling at $20 \mathrm{~W}$ ) concluded

142 the combined CPET session.

143

\section{MODEL SIMULATIONS}

145 Simulations are widely used in various fields of science and engineering because conducting physical

146 experiments is too costly, or highly time-consuming, or even impossible in some cases (28). In the

147 case of CPET in CF patients, there are also ethical considerations, since the test adds to the treatment

148 burden many children and adolescents with CF already face.

150 Often, a primary goal of using model simulations is to perform quantitative studies such as uncertainty quantification or sensitivity analysis. Such studies are crucially important in biomedicine, since there exists significant variation both between and within patient groups. Through understanding and quantification of the uncertainty within the mathematical models, outcomes of patient-specific interventions can be better predicted. However, such investigations require a large number of runs that makes it impractical if each run takes more than a few seconds. To cope with this difficulty, one can use emulators, also known as surrogates, or metamodels or response surfaces (29). These provide a fast approximation of the input/output relation governed by the underlying simulator. The most important classes of surrogate models have been described elsewhere (30-32). 
160 The surrogate model employed in this study is based on Gaussian processes (GP), which have

161 become increasingly popular over the last decade (29). GPs have been used in a wide range of

162 applications from wireless communication, to obtain position estimates for a mobile user (33);

163 metallurgy, to model the development of microstructure (34); and in biology, to describe gene

164 regulatory processes and cell growth (35-37).

166 The data analysis was performed using Python (Anaconda Software Distribution. Version 2-2.4.0.

167 Continuum Analytics, 2016. URL https://continuum.io) and MATLAB and Statistics Toolbox

168 Release 2016b, The MathWorks, Inc., Natick, Massachusetts, United States. The GP model simulator

169 was implemented in R (R Core Team (2013). R: A language and environment for statistical

170 computing. R Foundation for Statistical Computing, Vienna, Austria. URL http://www.R-

171 project.org/.)

172

\section{RESULTS}

\section{Data Analysis}

175 To facilitate understanding, we first plot in Figure 1(a), raw data displaying the performance of the

176 participants. The work rate for each participant is increased at a rate that is either, a) dependent on

177 their performance in previous tests, or b) when a prior test is unavailable, at a rate that is predicted to

178 elicit exhaustion in approximately ten minutes (26). This is done in order to keep the expected

179 duration of the test comparable to other participants. Note that this means that the total energy

180 expended by a given participant is not based on the duration of the test alone. In Figure 1(b), we

181 show how participant age affects overall test performance. We observe a correlation between the two:

182 the worst performing participants tend to be the youngest, but this effect is insignificant at older ages.

183 The colour coordination used in this figure (red-worst performance $\rightarrow$ blue-average performance

$184 \rightarrow$ green-best performance) will be used throughout the remainder of this section, where performance

185 is quantified by the total energy transferred during the test. 


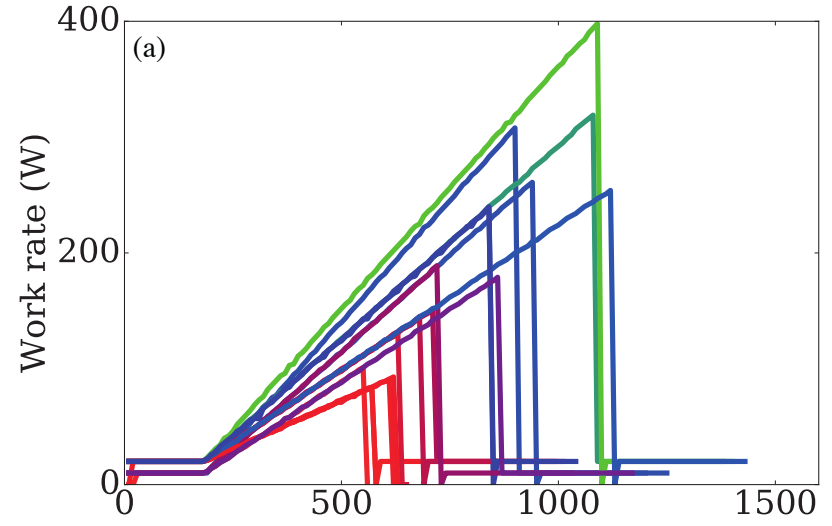

Time (s)

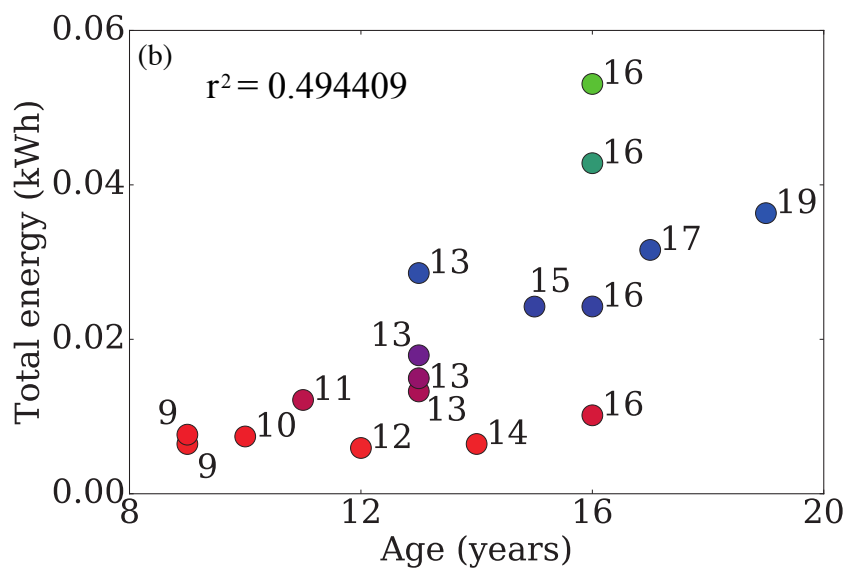

187 Figure 1: (a) The work rate for each participant is increased at a rate dependent on their past

188 test performance. (b) Participant age is correlated with test performance for young 189 participants, but not for older ones.

191 In Figure 2, we plot the ratio of $\dot{\mathrm{VO}}_{2}$ over total ventilation ( $\dot{\mathrm{V} E}$ ) with respect to time. The markers on 192 each of the time traces indicate the time of volitional exhaustion for that participant. There are two 193 features that stand out from this figure. Firstly, participants who perform better have higher $\dot{\mathrm{V}} \mathrm{O}_{2} / \dot{\mathrm{VE}}$ 194 ratios, suggesting that their oxygen uptake is more efficient than their poorer performing 195 counterparts. Secondly, in the recovery phase of the test (5 minutes following volitional exhaustion) better performing participants exhibit a sharp decrease in $\dot{\mathrm{V}}_{2} / \dot{\mathrm{VE}}$, which is not observed in the poor performance group. Again, this suggests a more efficient utilisation amongst the former group and that exhalation of $\mathrm{CO}_{2}$ is perhaps more significant to total breathing following the test.

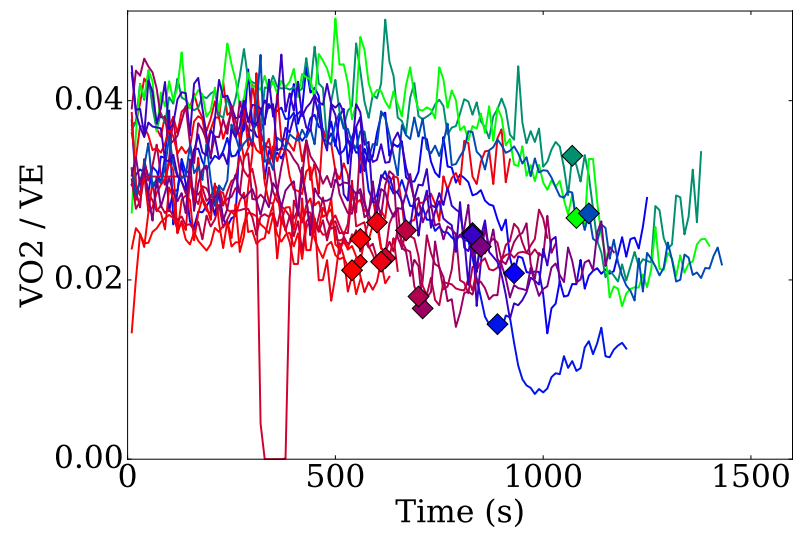

Figure 2: Ratio of oxygen utilisation and total breathing throughout the test. Markers indicate the volitional exhaustion times for each participant. 
203 We next examine the effect that breathing patterns have on participant performance. Two classical 204 prognostic measures used for patients with cystic fibrosis are the forced vital capacity (FVC), and the 205 forced expiratory volume in one second $\left(\mathrm{FEV}_{1}\right)$. These measures have been shown to be well correlated with mortality and overall fitness of CF patient groups (38-40). In Figure 3, we demonstrate how these metrics are correlated with performance in the CPET test. In Figure 3(a), we observe good correlation between FVC and the maximum tidal volume (TV) of breathing achieved throughout the test. This is unsurprising since participants are likely to be trying to maximise their breathing depth close to their exhaustion point. However, notice that, although the group with low FVC performed poorly, this measure was unable to separate other participants. Figure 3(b) reiterates this result and also highlights the high correlation between $\mathrm{FEV}_{1}$ and $\mathrm{FVC}$.

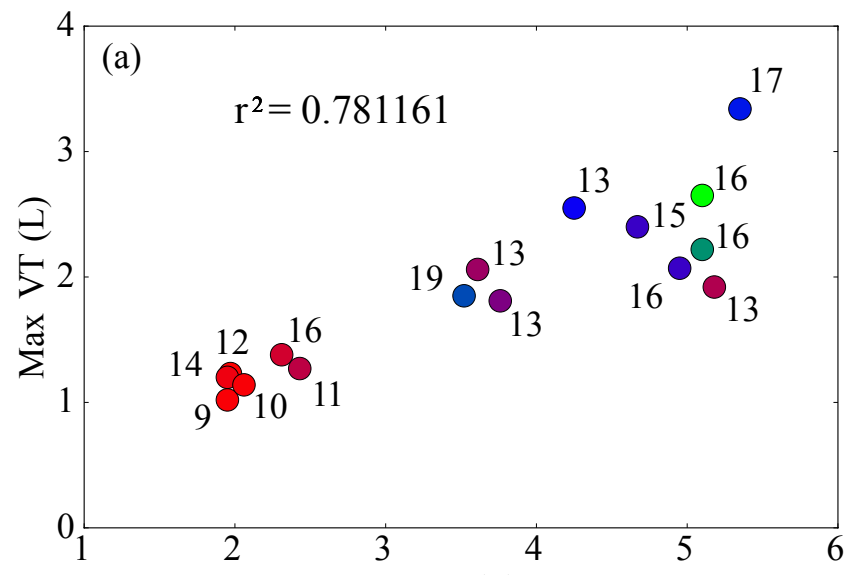

FVC (L)

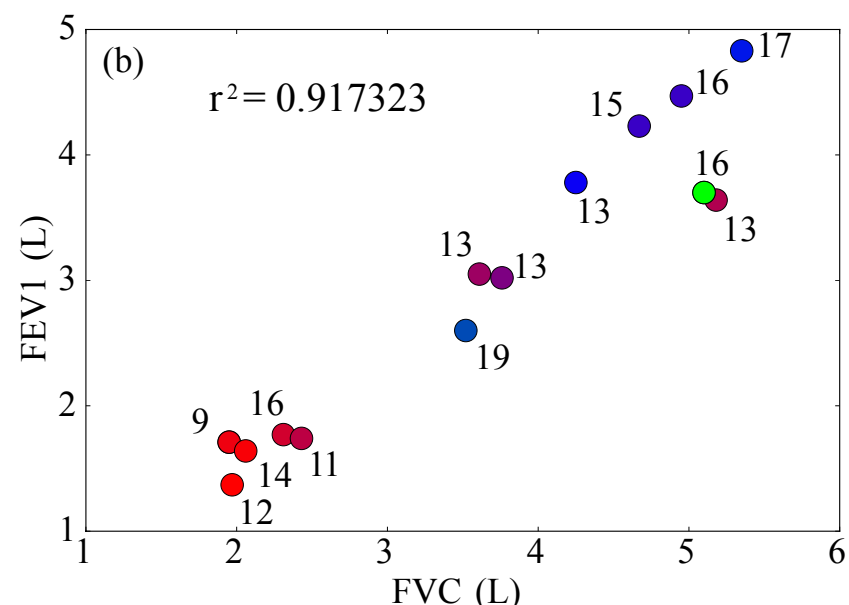

FVC (L)
213

214

215

216

Figure 3: (a) Correlation of $\mathrm{FEV}_{1}$ with the maximal tidal volume achieved throughout the test. (b) Correlation between FEV 1 and FVC is high. Note that, although FVC and FEV 1 are good predictors of poor test performance, they are unable to distinguish better performing participants.

In order to better classify the performance of the participants, we must instead look for other factors. In Figure 4, we present the total breathing rate against the oxygen consumption throughout the test. In Figure 4(a), we find a strong relationship between test performance and respiratory pattern. Note that the curvature of the graphs suggests that an exponential fit, rather than a linear one, is most appropriate for these data. In order to test this, we take logarithms of the data and perform a linear regression, ignoring the first 180 s of the test since participants are here in the warm up phase (work rate is not increasing) and the final 60 s of the data prior to volitional exhaustion, since participants pass their respiratory compensation point, inducing hyperventilation and erratic breathing. The 
results of the fit are shown in Figure 4(b) and we can see more clearly the association of

228 performance on breathing pattern.
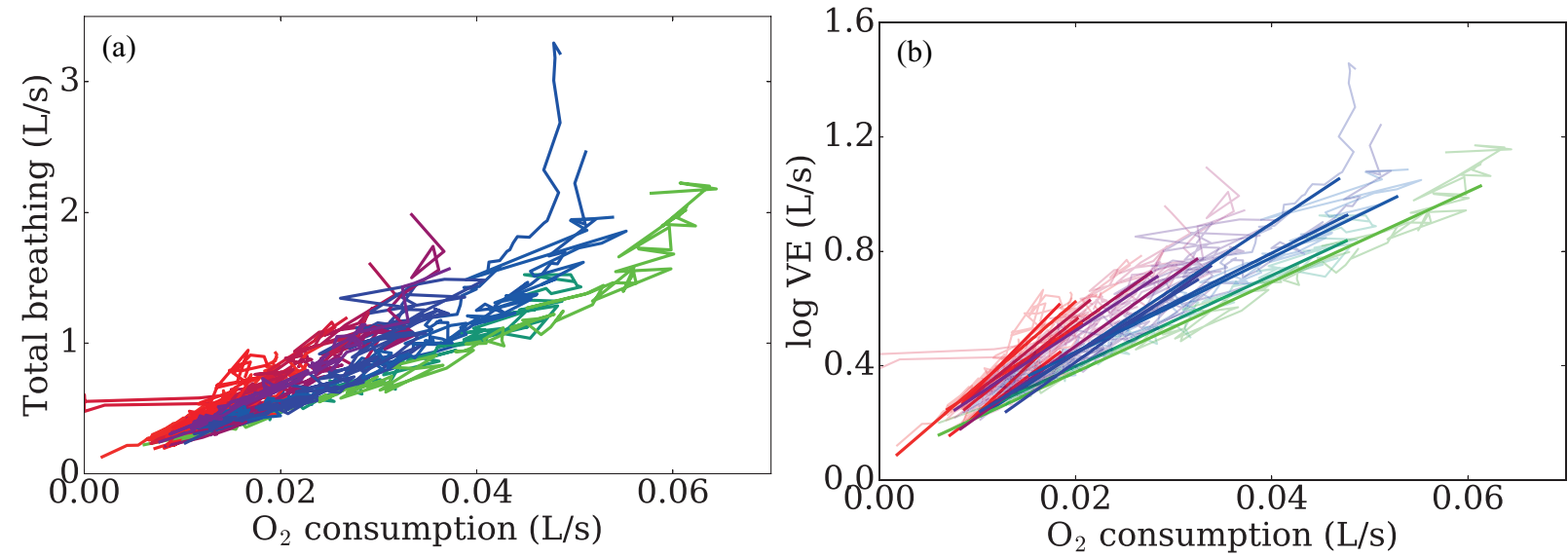

Figure 4: (a) Total ventilation plotted against oxygen utilisation. We observe that breathing pattern is strongly correlated with test performance. (b) Exponential curves are fitted through

From the fitted curves, we can further explore the dependence of breathing patterns on performance.

Firstly, in Figure 5(a), we plot the slope of the fitted curve against the total energy transfer. We find that the slope of the curve of $\log \dot{\mathrm{V} E}$ against $\dot{\mathrm{VO}}_{2}$ alone does not capture all of the variation in energy, which is highlighted by the low R-squared value (0.68). Instead, we plot in Figure 5(b) the oxygen consumption at a fixed rate of breathing against the total energy. Here, we find a good characterisation of the overall performance, with a much higher R-squared value (0.86), confirming that those who utilise oxygen more efficiently perform better.
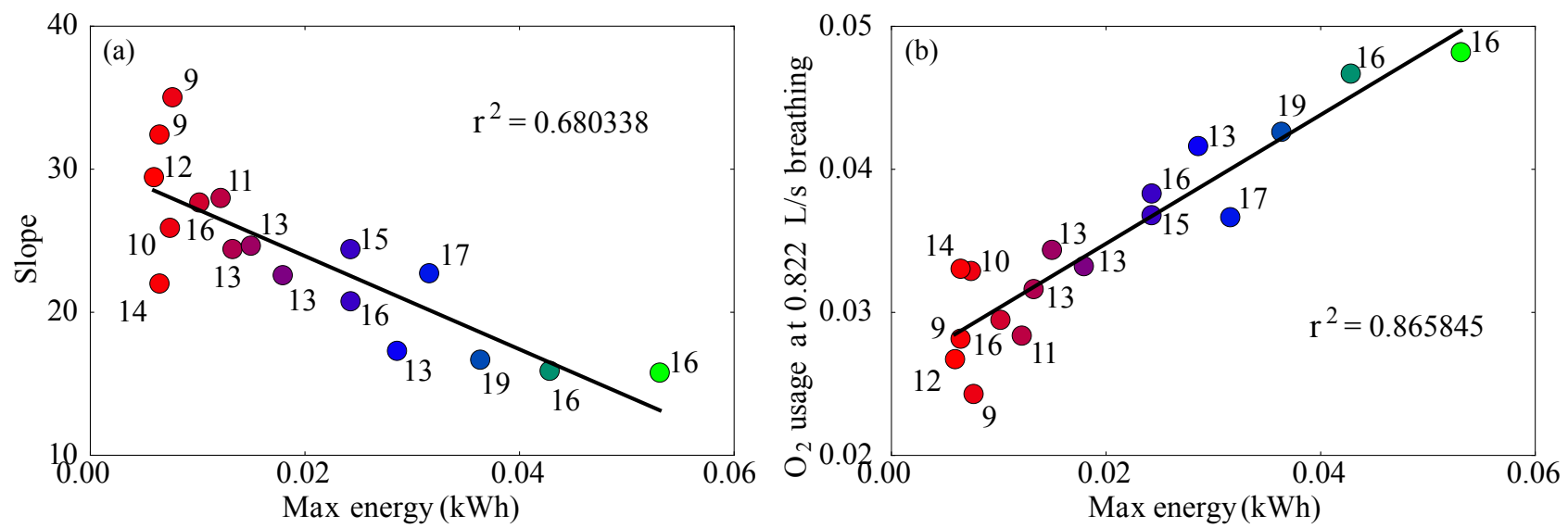

Figure 5: Slope of the fitted curves $\left(\log \dot{V} E\right.$ against $\left.\dot{V} O_{2}\right)$ from Fig. 4(b) plotted against the total 
between performances. (b) By instead plotting the oxygen consumption at a fixed rate of breathing, we better capture differences in performance.

248 Next, we examine the specific patterns of breathing exhibited by the participants, in particular, 249 focussing on breathing depth and frequency. Initial characterisations of these patterns seem to 250 provide little information, as indicated in Figure 6(a). However, when we now also include dependence of oxygen consumption, we find a near perfect classification of participants into the lowest performing groups, the best performing groups and the middle group. These data are displayed in Figure 6(b). Note that in this figure, the trajectories appear to be evolving on a planar manifold, suggesting significant co-dependence between these three variables.
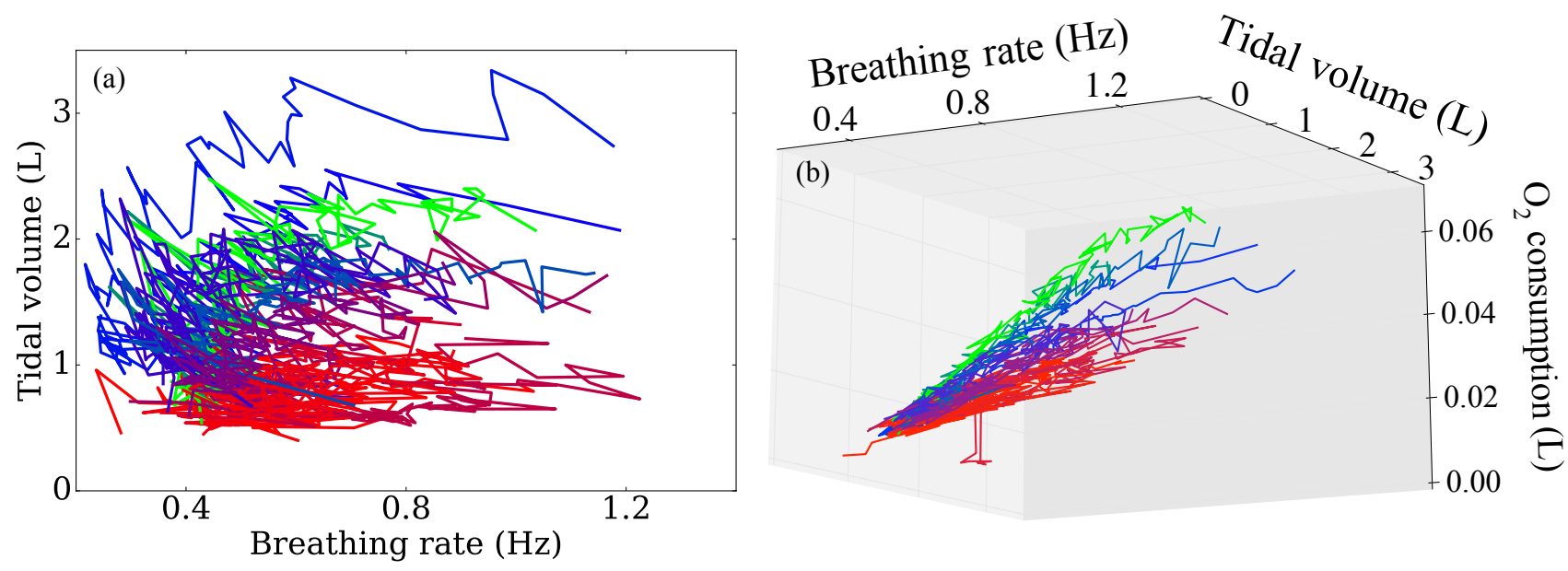

Figure 6: (a) Breathing patterns subdivided into the breathing rate and tidal volume. These data appear uninformative for predicting test performance. (b) With the additional inclusion of the oxygen consumption at a fixed rate of breathing, we find that these variables now almost perfectly capture variation in participant performance.

263 Given that there appears to be co-dependence between the variables used in Figure 6(b), a sensible next step is to use principal component analysis (PCA) to account for these dependencies. By projecting the data onto their principal components, we show in Figure 7 how well these capture the variation in participant performance. Given that there are only three independent variables in our analysis, it is natural to use spherical polar coordinates to show how these quantify performance. The first of these components, $\theta$, captures over $90 \%$ of the variation in performance (Figure 7a), as does 
the normal component in the direction of breathing frequency (Figure 7b). These results further indicate the importance of breathing frequency, together with co-variation of oxygen consumption and tidal volume as predictors of test performance.

272

273

274

275

276

277

278

279

280

281

282

283

284

285
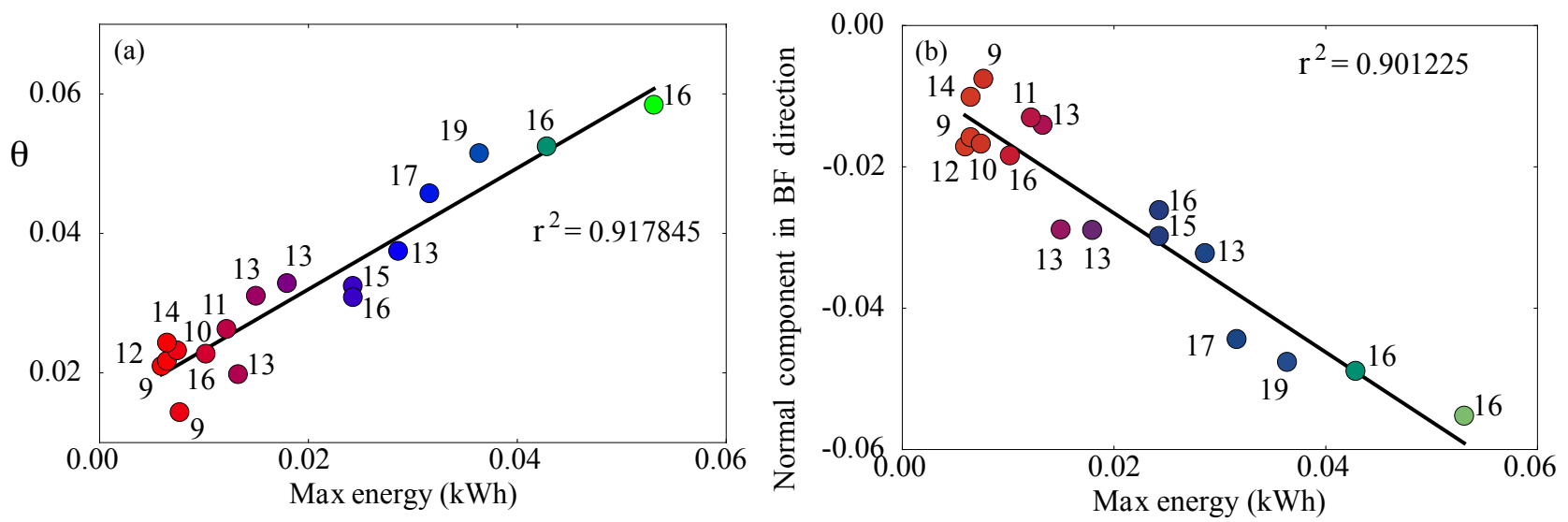

Figure 7: First of the principal components obtained via PCA accounts for over 90\% of the variation in test performance. (b) Similar levels of variance are accounted for by taking only the normal component of the first principal component in the breathing frequency direction.

\section{Gas Exchange Threshold}

Under steady state levels of exercise, the metabolic rate of production of $\mathrm{CO}_{2}$ is assumed to be proportional to the utilisation rate of $\mathrm{O}_{2}$ via the cellular respiratory quotient, since (after the initial rest-work transition) adenosine triphosphate (ATP) is replenished primarily via aerobic metabolism pathways. As the work rate increases, this pathway becomes unable to supply sufficient ATP to satisfy the required amount of energy and anaerobic pathways have to contribute to overcome the shortfall. In doing so, they increase the levels of waste products, such as lactate and also increase the overall production rate in $\mathrm{CO}_{2}$. The point at which this occurs is known as the anaerobic threshold (AT) or sometimes referred to as the lactate threshold. However, this is difficult to directly measure in vivo during exercise, and as such the GET is utilised as a non-invasive surrogate of the AT (41).

The time at which participants cross the anaerobic threshold is well correlated with overall performance, as shown in Figure 8(a), due to the fact that the anaerobic pathways are less efficient at producing ATP and because build-up of lactate contributes significantly to fatigue. One of the major contributing factors in defining AT is $\dot{\mathrm{V}} \mathrm{O}_{2 \max }$, since this is indicative of the limit of the rate of oxidative phosphorylation. It thus comes as little surprise that $\dot{\mathrm{V}} \mathrm{O}_{2 \max }$ is the best single predictor of CPET performance, as shown in Figure 8(b). 

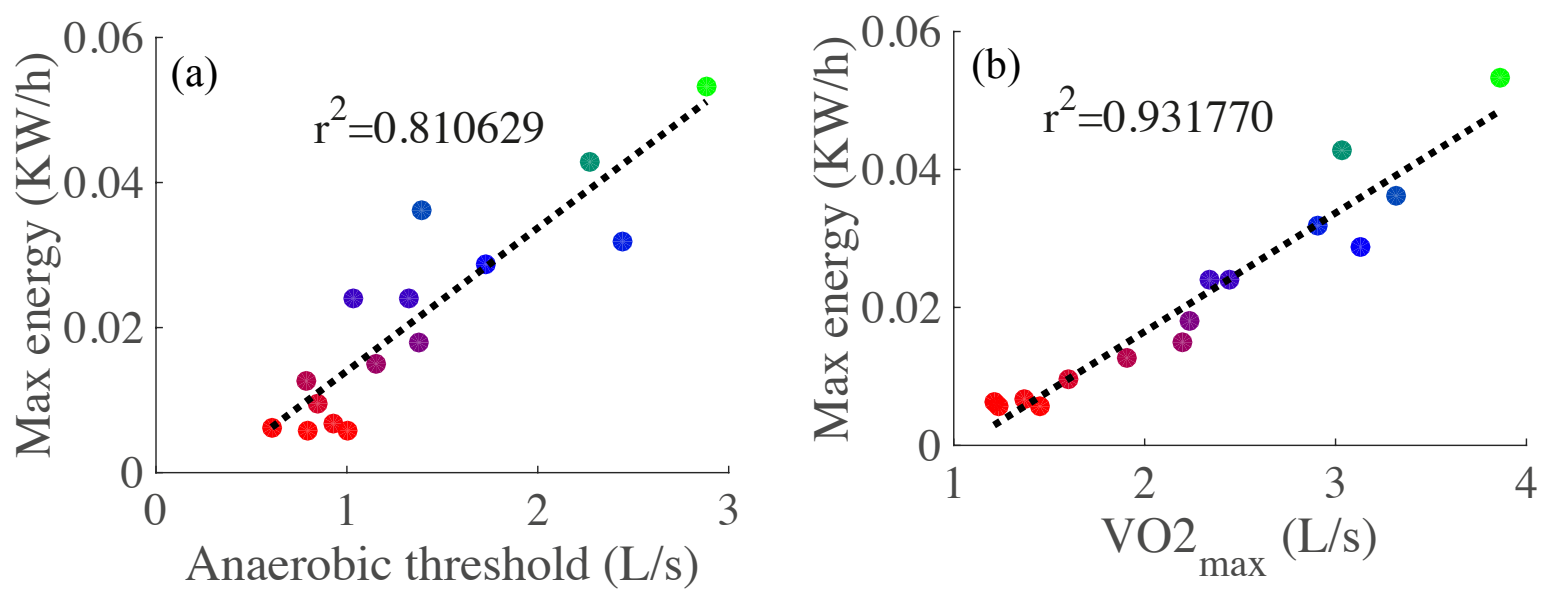

Figure 8: (a) Quantifying the relationship between the anaerobic threshold and overall test performance. Anaerobic thresholds were calculated using an automated procedure based on previous methods (41) (b) $\dot{\mathrm{V}} \mathrm{O}_{2 \mathrm{max}}$ is the best single predictor of overall test performance.

A Gaussian Process (GP) defines a probability distribution over functions where the true function is considered as a particular sample path. In our modelling, we attempt to describe the influence of breathing patterns and $\dot{\mathrm{VO}}_{2}$ on the AT, since this is shown to correlate well with overall test input variables comprising: baseline breathing rate and tidal volumes, and $\mathrm{O}_{2}$ consumption rates at a fixed ventilation rate, $\mathrm{FVC}, \mathrm{FEV}_{1}$, and the rate of changes in breathing rate and tidal at exercise onset, using the slopes calculating in Figure 4(b). Thus, we have an output variable dependent on seven input variables, which we conveniently store in a vector $\mathbf{x} \in D \subset \mathbb{R}^{\boldsymbol{d}}$, with $d=7$. We now assume that there exists a 'true' function $f: D \rightarrow \mathbb{R}$, such that $\mathrm{AT}=f(\mathbf{x})$.

A GP is fully specified by its mean, $\mu$, and covariance $K$, which are both functions of the input variables: $\mu=\mu(\mathbf{x}), K=K\left(\mathbf{x}, \mathbf{x}^{\prime}\right)$. Specifically, if $Y$ is a GP, then we write:

$$
Y \sim G P(\mu, K): \quad \mu=\mathrm{E}[Y(\mathbf{x})], \quad K\left(\mathbf{x}, \mathbf{x}^{\prime}\right)=\operatorname{Cov}\left(Y(\mathbf{x}), Y\left(\mathbf{x}^{\prime}\right)\right), \quad \mathbf{x}, \mathbf{x}^{\prime} \in D
$$

316 The above can be regarded as prior distribution over function spaces. This can be seen more clearly

317 in Figure 9(a). In this subfigure, which shows a generic example of a GP, the bold red line is the 318 'true' function $f$. Note that the true function is unknown - our aim is to construct a model that 
approximates it. The thin grey lines are sample paths of a GP with a zero mean prior and a constant prior covariance function. For exposition purposes, the example plot is restricted to the case $d=1$, but the approach is unchanged for $d>1$. In principle, $\mu$ could be any function, for practical purposes, polynomial regressions are common choices. The choice of covariance function reflects our prior belief about the structure of $f$ (such as the level of smoothness) and therefore has a crucial influence on our modelling. A typical choice for the covariance function is the squared-exponential kernel, given by:

$$
K\left(\mathbf{x}, \mathbf{x}^{\prime}\right)=\sigma^{2} \prod_{i=1}^{d} \exp \left(-\frac{\left|x_{i}-x_{i}^{\prime}\right|^{2}}{2 \Phi_{i}^{2}}\right)
$$

Here, $\sigma$ controls variability of $Y$ along the y-axis while $\Phi_{i}>0, i=1, \ldots, d$, scale the distance measure for each input dimension. A more thorough exposition of common choices for the covariance function may be found in (42).

Thus far, we have defined the prior distribution for the GP. It is clear from Figure 9(a) that, in general, prior distributions are unlikely to provide a good approximation to the true function $f$. We can incorporate data (or evaluation of $f$ at specific points), with our prior distribution to give a posterior distribution, following a Bayesian framework. The resulting posterior distribution of the

Let $\mathbf{y}=\left\{f\left(\mathbf{x}^{1}\right), \ldots, f\left(\mathbf{x}^{n}\right)\right\}$ be a set of function evaluations at $n$ locations $\mathbf{X}=\left\{\mathbf{x}^{1}, \ldots, \mathbf{x}^{n}\right\}$. Here, posterior distribution of $Y(\mathbf{z}) \mid \Omega$ has a normal distribution with the following mean, $m$, and variance,

$$
\begin{aligned}
& m(\mathbf{z})=\mu(\mathbf{z})+K(\mathbf{z}, \mathbf{X})^{\top} K(\mathbf{X}, \mathbf{X})^{-1}(\mathbf{y}-\boldsymbol{\mu}), \\
& s^{2}(\mathbf{z})=K(\mathbf{z}, \mathbf{z})-K(\mathbf{z}, \mathbf{X})^{\top} K(\mathbf{X}, \mathbf{X})^{-1} K(\mathbf{z}, \mathbf{X}),
\end{aligned}
$$

where ${ }^{\mathrm{T}}$ denotes the transpose operator, ${ }^{-1}$ is the inverse operator and $\boldsymbol{\mu}=\mu(\mathbf{X})$ is the vector of the 
and the covariance matrix between the observations. Figure 9(b) shows an example of incorporating sample points to update the prior distribution shown in Figure 9(a). In this generic example, the function $f$ is evaluated at five distinct values of $\mathbf{x}$, and the mean and variance of the GP are updated using (1)-(2).

(a)

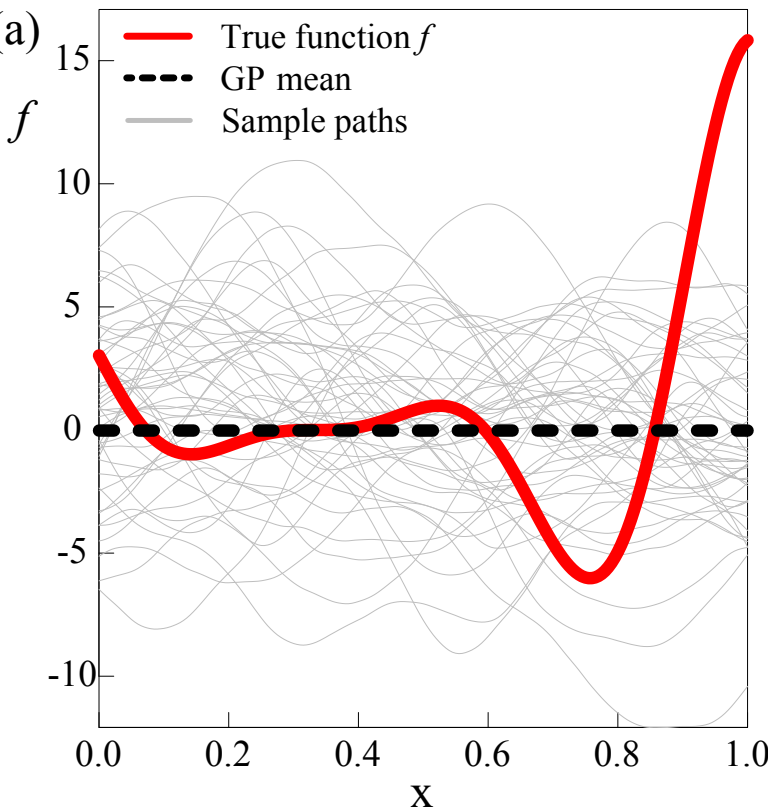

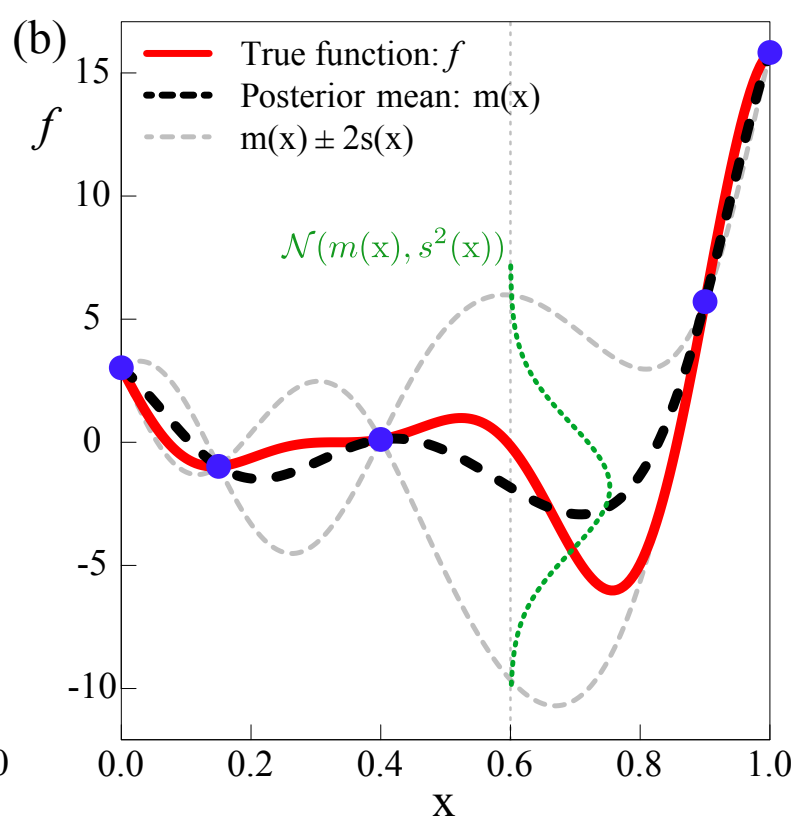

Figure 9: GP is a probability distribution on function spaces. The thick solid red line is the true function $f$ and the thick dashed black line is the GP mean. (a) Thin grey lines show sample paths of a prior GP whose mean is zero. (b) Blue bullets indicate five data points sampled from $f$. The GP mean and covariance are updated using these sample points. The thin grey dashed lines show $m(\mathbf{x}) \pm 2 s(\mathbf{x})$.

At the evaluated points, indicated in blue (colour online), the true value of $f$ is known and so the variance of the GP at these points vanishes and $\mu(\mathbf{X})=f(\mathbf{X})$. In between these points, the variance increases, dependent on the distance from an evaluated point. The mean of the GP, shown by the thick black dashed line now approximates the true function much more closely (recall that the prior mean function in Figure 9(a) was zero everywhere), and matches exactly at the evaluated points. The approximation can be further improved by incorporating more data (function evaluations), particularly around those input values for which the variance is high. Thus, as more data becomes available, the model is iteratively improved. 
371 In our simulator, we have used the AT as our output (dependent) variable. Another choice for this

372 could be the performance in the CPET or $\dot{\mathrm{VO}}_{2 \max }$, since these are the primary biomarkers for gauging

373 aerobic fitness. However, the use of the GET has been shown to have high agreement with the lactate

374 threshold (another surrogate for the AT), and related to disease severity in CF (43). Furthermore, as

375 reported in Figure 8(a), the AT location for a given patient correlates well with their overall

376 performance in this test. Furthermore, by constructing a predictive model to approximate the AT

377 values for a patient, we can hope to further extend this to identify contributions of aerobic and

378 anaerobic pathways in supplying ATP to meet the demand imposed during the exercise test.

380 The initial exploration of results highlighted that both ventilation parameters and metabolic rates of

$\mathrm{O}_{2}$ consumption were the primary factors influencing test performance. It is clear that $\dot{\mathrm{VO}}_{2}$ should play a significant role in determining the AT location, since it is a proxy for oxidative phosphorylation which is the main pathway for ATP synthesis in steady state exercise. As a measure of oxygen uptake efficiency in our model, we use the oxygen consumption rate at a fixed total ventilation rate (that being $0.822 \mathrm{~L} / \mathrm{s}$ ) as an input (independent) variable for each patient.

387 There are a number of ventilatory input variables incorporated in our simulator. Given their potential importance as clinical biomarkers, highlighting the limitations of lung capacity and function, we include $\mathrm{FVC}$ and $\mathrm{FEV}_{1}$ as input (independent) variables. During the aerobic exercise test, participants spend three minutes cycling at a minimal work rate, over which we quantify their baseline breathing capture the dynamics response associated with the exercise, the rates of change of breathing frequency and tidal volume are calculated, based on the fits obtained in Figure 4(b). The rates of change of these ventilatory variables indicate how participants respond to changes in workload and were shown in Figure 6(b) to discriminate between participant performances. Moreover, differences in rates of change of breathing frequency and tidal volume have previously been shown to be significantly different between control groups and CF groups (44), suggesting that these are potentially key biomarkers for assessing aerobic fitness in patients with $\mathrm{CF}$.

\section{GP Emulator Performance}

401 The GP emulator was constructed using the data presented above, with AT calculated using 402 previously described methods (41). These data were used to train the emulator. For the prior 403 distributions, a first order polynomial regression was used for the mean, whilst a squared-exponential 
404 kernel with $\sigma$ and $\Phi$ provided via maximum likelihood estimation was used as the prior covariance.

405 Since in this pilot study, we have a small number of participants, we use leave-one-out cross-

406 validation to assess the accuracy of our emulator, that is, for each patient, we use the emulator trained against the remainder of the sample points to approximate the AT value for that patient, given their

408 input variables. The results of this are presented in Figure 10(a), in which we also plot the 95\%

409 confidence intervals, with relative percentage errors demonstrated in Figure 10(b).

410

411

(a)

412

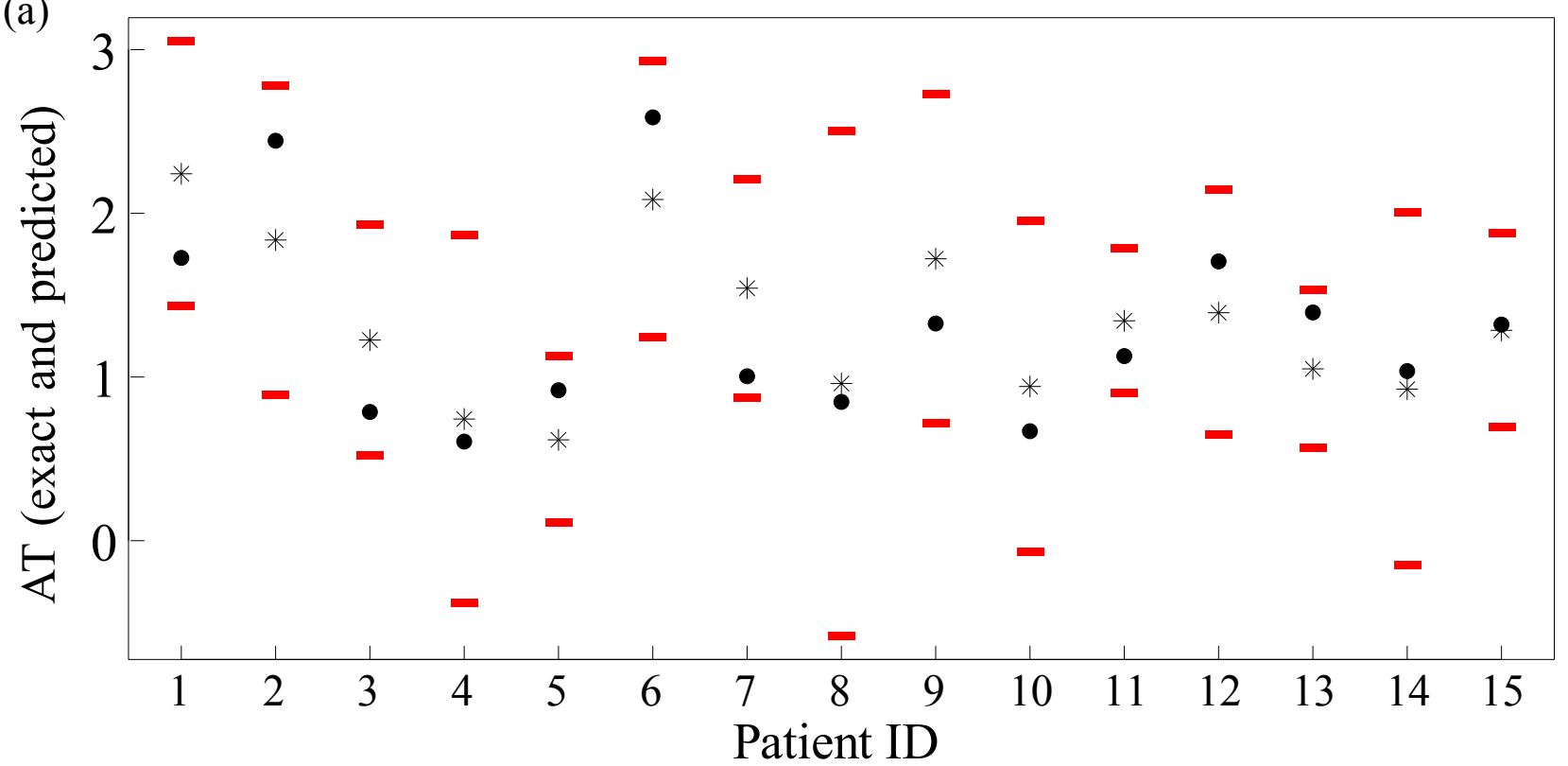

(b)

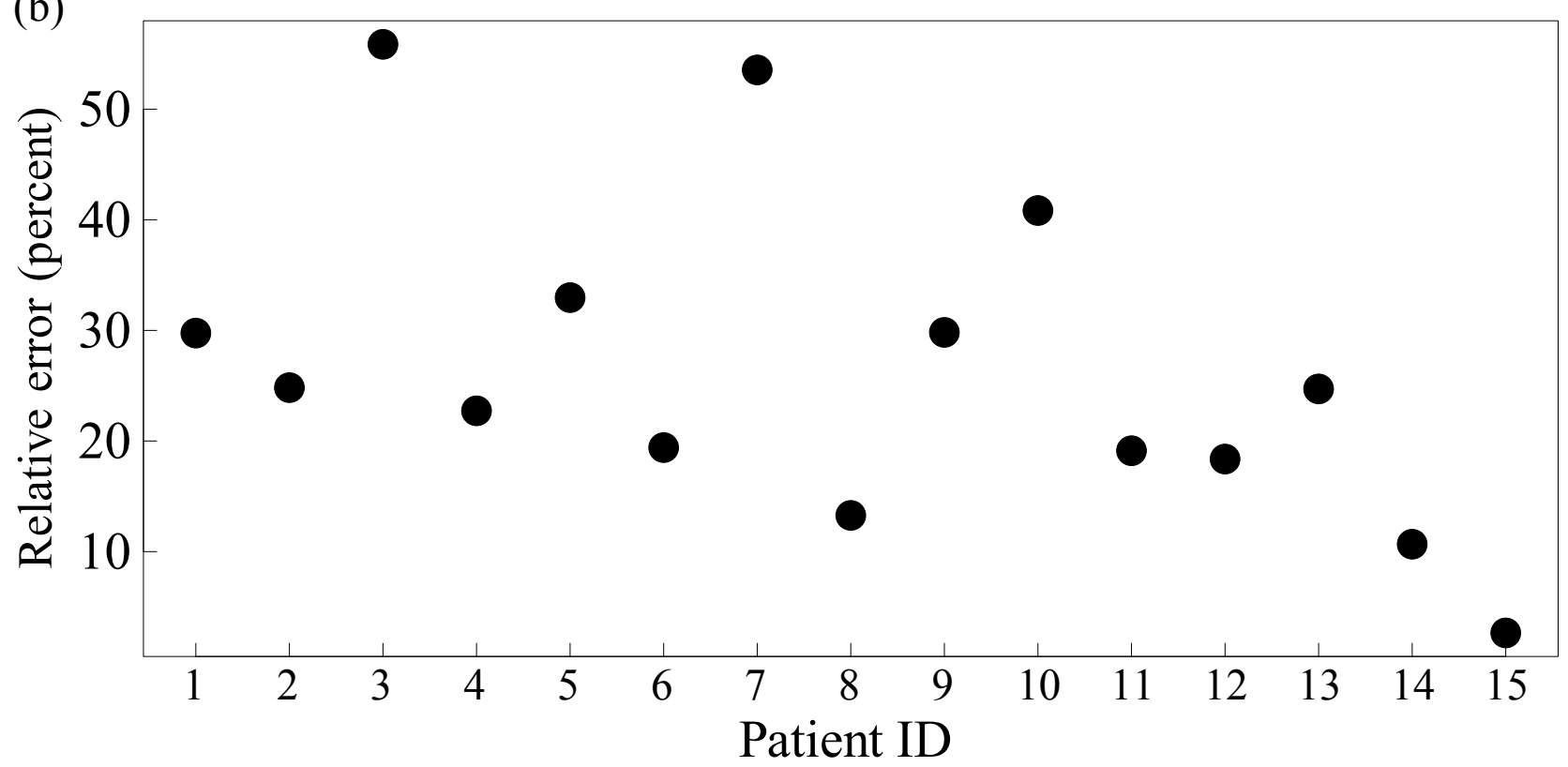


416 Figure 10: (a) Predictions (asterisks) vs. exact values (bullets). Red bars show the 95\%

417 confidence intervals, $\boldsymbol{m}_{\mathbf{i}}\left(\mathbf{x}_{\mathbf{i}}\right) \pm \mathbf{1 . 9 6} s_{i}\left(\mathbf{x}_{\mathbf{i}}\right)$ around the predicted value, where $\boldsymbol{m}_{\mathrm{i}}$ and $s_{\mathbf{i}}$ are GP 418 prediction mean and standard deviation based on all but the $i$ 'th data point. (b) Relative 419 prediction error expressed as a percentage of the true value $f\left(\mathbf{x}_{\mathbf{i}}\right)$.

421 The emulator has reasonable accuracy, in spite of the small sample size. In general, for high accuracy 422 in GP emulator, the number of sample points (corresponding to the number of patients in our case) 423 should be at least ten times larger than the number of input variables (45). Clearly, there is a need to acquire further data points to improve the predictive capabilities of the emulator. For each patient, the 95\% confidence interval around the predicted point contains the true value. Moreover, the relative errors for many patients is small (considering the low sample size), though we note that some patients, (3 and 7), the error is high. This highlights a need to extend this study to include more data to refine estimates around these points, particularly to deal with the high variability of lung function parameters in this patient group (46).

\section{DISCUSSION}

432 Our primary modelling aim is to eventually use the model to evaluate how CF impairs exercise tolerance relative to increasing ventilatory and metabolic demands. Our predictive model could also be used to evaluate therapies and their effect on exercise performance. Ultimately, we hope that this will form a series of steps to design better exercise treatment that is tailored to specific individual needs relative to patient's treatment therapies, a treatment modality that is affordable, and personalised (47).

The data analysis and modelling results have highlighted the dependence of CPET performance on variables associated with ventilation and metabolic rates of $\mathrm{O}_{2}$ consumption. Whilst these observations are, in themselves, not novel, we believe that this is the first attempt to mathematically model the relationship between ventilatory dynamics, $\mathrm{VO}_{2}$ and performance in CPET within a group of children and adolescents with CF. Whilst it is clear that there is much work to be done in this area, we hope that this will serve as a starting point for improved modelling, not only in the arena of GP emulators, but also in the domain of mechanistic modelling, which we shall describe briefly. 
At present, the GP model is conditioned on specific data points for each patient. An improvement to the GP could be made by instead conditioning with respect to distributions. Given that repeated tests are often performed for the same individual, so that multiple sample points are provided for each participant, we can consider a fit to a probability distribution capturing the variability in the identified variables. This approach has advantages compared to standard GP models, such as avoiding problems associated with overfitting and regularisation (which is important for the inverting illconditioned covariance matrices that often arise during the application of (1)-(2), (48).

\section{Perspectives for mechanistic modelling}

457 In order to better understand and characterise the difference between performances, it would be 458 extremely useful to construct and simulate a mechanistic mathematical model, based upon on ordinary differential equation (ODE) framework, describing the relationship between the cardiopulmonary system and the metabolic dynamics of skeletal muscle. By describing the relationships between different organ-level systems, the model would be able to identify the patientspecific rate-limiting factors defining aerobic fitness. Moreover, analysis of the model could be used to suggest treatment strategies to improve these factors and thus predict how patients will improve under such regimes.

At the individual organ level, there are a plethora of models describing individual dynamics of the level of the heart (49-52), lung (53-56) and systemic metabolic demand (57-63). There also exist a number of models describing such interactions between cardiopulmonary and metabolic systems (6468 ) in a variety of settings, including heart failure and mechanical ventilation. A core feature in all of these models is the nonlinear interaction between the constituent model compartments that encompass the distinct tissues. An important consequence of this is that the model must be studied as whole, in an integrated fashion, to truly understand the body's response to exercise.

474 With respect to the present question, there are a number of limitations of the existing modelling approaches. Most significantly, none have been designed with either an adolescent, or a CF patient group in mind, and the nuances of these patient groups will have to be factored into to any model development. In particular, these models have relatively simple, empirical models to describe changes in ventilation, which may not capture breathing dynamics of our patient group well. Moreover, to the best of our knowledge, no model considers the changes in ventilation separated into 
breathing frequency and depth that have been shown by us and others (44) to be critical to overall test performance.

In our analysis, we have demonstrated that the AT or GET location is a critical factor in determining overall patient aerobic fitness. Many of the mathematical exercise models describe only steady-state exercise, in which aerobic pathways meet most of the ATP demand (64-68). As such, these models are inadequate to capture the dynamics we describe here. Another common topic of study is the dynamic response at exercise onset, which again, does not meet the current need to describe the AT crossing point (69-72).

Of the mathematical models that describe the contribution of anaerobic pathways to ATP production, some assume that the shortfall in meeting ATP demand via oxidative phosphorylation is met entirely by anaerobic pathways (73), yet this is clearly not so, since ATP levels in skeletal muscle postfatigue brought about by anaerobic metabolism are generally phenomenological in nature, and it is difficult to quantify these models against real patient data $(73,75,76)$, and these models mostly fall outside the arena of ODE-based modelling and so dynamical properties are difficult to infer from them.

Developing a mathematical model to describe the integrated behaviour of all of the relevant organs, whilst remaining biophysically plausible, but without requiring excessive or invasive parameterisation is a difficult task. The proposed model should include descriptions of the cardiovascular system, the ventilatory system and simple models of metabolism at the tissue level. Specifically, dynamic variables should include alveolar, arterial, venous and tissue level partial pressures/concentrations of $\mathrm{O}_{2}$ and $\mathrm{CO}_{2}$, cardiac output, ventilation and metabolic rates oxygen utilisation and $\mathrm{CO}_{2}$ production. Partial alveolar gas pressures can be linked to data collected during the test, and the work rate can then be provided as inputs to the model. Note that these variables are similar to those included in previously defined models (64-68) and the aim is to extend these to describe the dynamics observed in patients with CF. The proposed model schematic is displayed in

511 Of critical importance to the overall model construction is the development of a simple, yet realistic

512 model of cellular metabolism, to overcome the issues discussed earlier. The model should respect the 
513 different metabolic processes that occur in the muscle tissue, in particular: glycolysis,

514 phosphocreatine breakdown and synthesis and oxidative phosphorylation, in a simplistic fashion that

515 is amenable to being fit to CPET data. Whilst there are models that describe the biochemical

516 reactions associated with these processes, and importantly, their stoichiometry $(58,61,63,67,77)$,

517 quantifying their associated rate constants in vivo is a near-impossible task, and so efforts must be

518 made to develop a model that incorporates the relevant metabolic dynamics whilst being simple

519 enough to be fit to data.

520

521 With knowledge of the integrated system, attempts can also be made to describe other important

522 exercise-based processes, such as lactate buffering and recycling (as a fuel source) $(61,78,79)$ and

523 the overall muscle fatigue brought about by the combination of all of these factors. Only by

524 systematically exploring the dependence of aerobic fitness of all of the factors described in this

525 section can we begin to understand the system in an integrated fashion.

526

527 


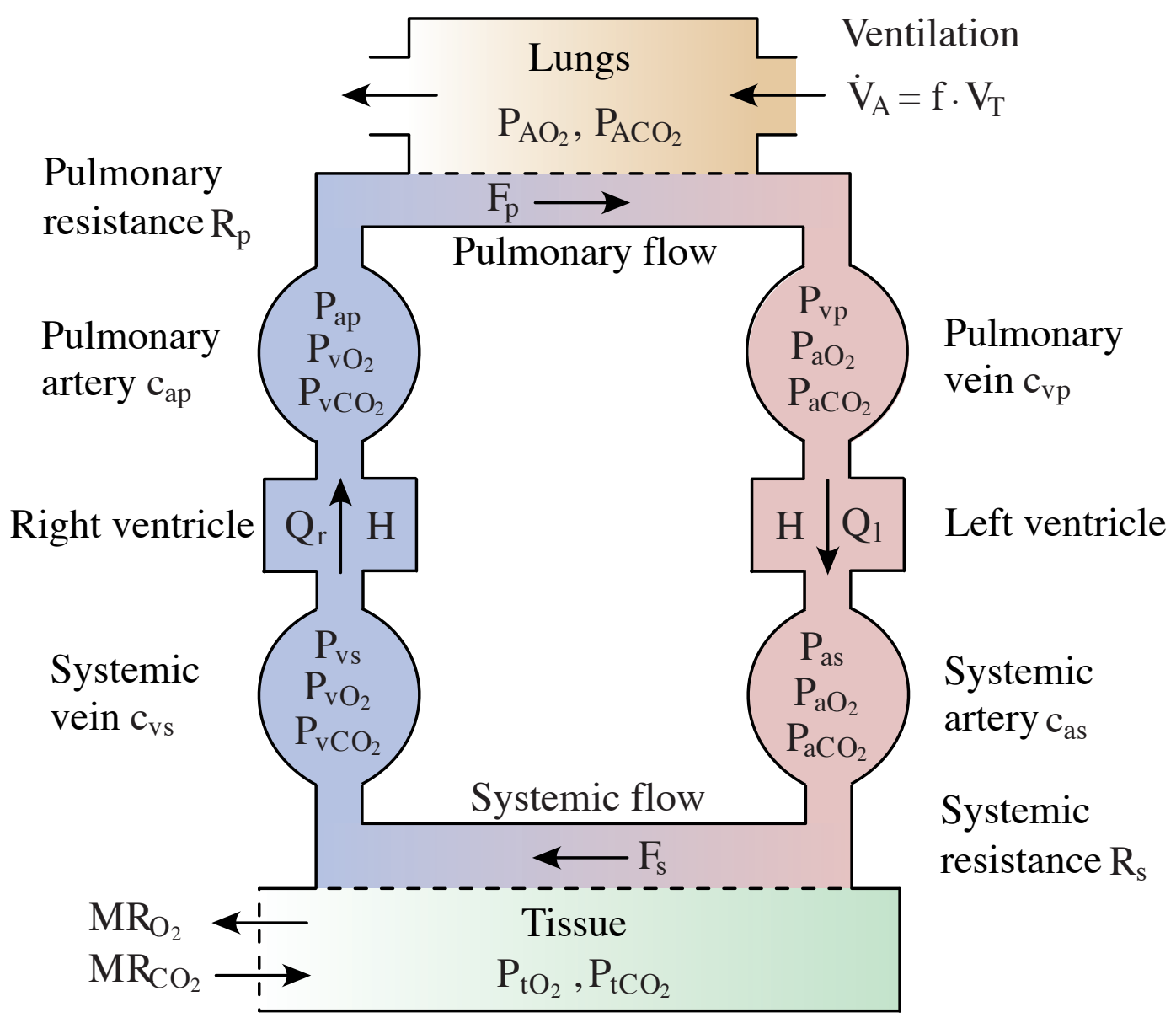

530 Figure 11: Schematic of the variables and processes in the proposed ODE-based mathematical model. Adapted from Timischl (1998) (66) and Batzel et al. (2005) (80).

\section{LIMITATIONS}

534 A limitation with the current study is the utilisation of a relatively small sample size, and this may be contributing towards aforementioned errors. Future studies should seek to utilise CPET collected annually in CF centres, to develop larger, multi-centre, samples whereby a uniform exercise protocol is utilised. Given that utilisation of CPET is now recommended and endorsed for regular use by international medical societies (81), and individual CF centres are reporting upon experiences of using CPET (82), large-scale utilisation of such data is a feasible target.

The findings presented may be derived from a smaller sample, and therefore the models presented are only preliminary results for this patient cohort; however, the study provides a unique examination

543 into the aerobic and anaerobic signatures of individual patients with $\mathrm{CF}$ in response to progressive 544 exercise. 
546 Finally, whilst this study provides an insight into metabolic process during exercise, future research and models must account for additional variables predictive of function and mortality (e.g. genotype, body composition, pancreatic sufficiency, infection status, exacerbations $(83,84))$ and co-morbidities (CF-related diabetes (85), pulmonary arterial hypertension (86)) existent within CF, notably those that may affect exercise tolerance.

\section{CONCLUSIONS}

553 Benefits of modelling include the ability to utilise existing data sets at a time when there are limited resources. There is also a call to reduce the burden on sick patients (EU directive). As models improve and the quality of fits to data are improved, the models can be used in a prognostic setting to predict potential improvements in aerobic fitness that may arise due to therapeutic intervention. Moreover, with proper mechanistic modelling of the primary organs affected in $\mathrm{CF}$, there exists the potential to optimise treatment for this patient group by identifying the limiting factors of aerobic fitness.

\section{CONFLICT OF INTEREST}

The authors declare that the research was conducted in the absence of any commercial or financial relationships that could be construed as a potential conflict of interest.

\section{AUTHOR CONTRIBUTIONS}

CAW conception, design of the work, data collection, analysis, interpretation, manuscript drafting, final approval of the version to be published, agreement to be accountable for all aspects of the work in ensuring questions related to the accuracy and integrity of any part of the work are appropriately investigated and resolved; KTA data interpretation, critical review of the paper, final approval of the version to be published, agreement to be accountable for all aspects of the work in ensuring questions related to the accuracy and integrity of any part of the work are appropriately investigated and resolve; OWT data collection, analysis, interpretation, final approval of the version to be published, agreement to be accountable for all aspects of the work in ensuring questions related to the accuracy and integrity of any part of the work are appropriately investigated and resolved; KW supervision of work organization and development, data analysis, interpretation, manuscript drafting, final approval of the version to be published, agreement to be accountable for all aspects of the work in ensuring questions related to the accuracy and integrity of any part of the work are appropriately investigated 
and resolved. HM construction of GP emulator, final approval of the version to be published,

579 agreement to be accountable for all aspects of the work in ensuring questions related to the accuracy

580 and integrity of any part of the work are appropriately investigate and resolved.

\section{FUNDING}

583 The present work was funded by an ISSF2 Wellcome Trust Seed Corn grant.

\section{ACKNOWLEDGMENTS}

586 We are grateful to the participants for volunteering their time to this project, especially the young patients with CF. KW was generously supported by the Wellcome Trust Institutional Strategic via grant EP/N014391/1.

\section{Supplementary Material}

592 Supplementary Material should be uploaded separately on submission, if there are Supplementary 593 Figures, please include the caption in the same file as the figure. Supplementary Material templates 594 can be found in the Frontiers Word Templates file.

595 Please see the Supplementary Material section of the Author guidelines for details on the different 596 file types accepted.

REFERENCES

601 1. Cystic Fibrosis Trust. UK Cystic Fibrosis Registry 2015 Annual Data Report. 2016.

602 2. Pérez M, Groeneveld IF, Santana-Sosa E, Fiuza-Luces C, Gonzalez-Saiz L, Villa-Asensi JR, 603 et al. Aerobic fitness is associated with lower risk of hospitalization in children with cystic fibrosis. 604 Pediatric Pulmonology. 2014;49(7):641-9.

605 3. Andréasson B, Jonson B, Kornfält R, Nordmark EVA, Sandström S. Long-term Effects of 606 Physical Exercise on Working Capacity and Pulmonary Function in Cystic Fibrosis. Acta Paediatrica. 607 1987;76(1):70-5. 
608 4. Orenstein DM. Exercise conditioning and cardiopulmonary fitness in cystic fibrosis. The

609 effects of a three-month supervised running program. Chest. 1981;80(4):392.

610 5. Salh W, Bilton D, Dodd M, Webb AK. Effect of exercise and physiotherapy in aiding sputum 611 expectoration in adults with cystic fibrosis. Thorax. 1989;44(12):1006-8.

612 6. Schneiderman-Walker J, Pollock SL, Corey M, Wilkes DD, Canny GJ, Pedder L, et al. A 613 randomized controlled trial of a 3-year home exercise program in cystic fibrosis. The Journal of 614 Pediatrics. 2000;136(3):304-10.

615 7. Nixon PA, Orenstein DM, Kelsey SF, Doershuk CF. The prognostic value of exercise testing 616 in patients with cystic fibrosis. New England Journal of Medicine. 1992;327(25):1785-8.

617 8. Wheatley CM, Wilkins BW, Snyder EM. Exercise is medicine in cystic fibrosis. Exercise and 618 Sport Sciences Reviews. 2011;39(3):155-60.

619 9. Stevens D, Oades PJ, Armstrong N, Williams CA. Exercise metabolism during moderate620 intensity exercise in children with cystic fibrosis following heavy-intensity exercise. Applied 621 Physiology, Nutrition, and Metabolism. 2011;36(6):920-7.

622 10. Williams CA, Stevens D. Physical activity and exercise training in young people with cystic 623 fibrosis: Current recommendations and evidence. Journal of Sport and Health Science. 2013;2(1):3962446.

625 11. Angelis A, Kanavos P, Lopez-Bastida J, Linertova R, Nicod E, Serrano-Aguilar P, et al. 626 Social and economic costs and health-related quality of life in non-institutionalised patients with 627 cystic fibrosis in the United Kingdom. BMC Health Services Research. 2015;15(1):428.

628 12. Stevens D, Oades PJ, Armstrong N, Williams CA. A survey of exercise testing and training in 629 UK cystic fibrosis clinics. Journal of Cystic Fibrosis. 2010;9(5):302-6.

630 13. Goodson HV, Gregoretti IV. Using Computational Modeling to Understand Microtubule 631 Dynamics. Methods in Cell Biology. 2010;95:175-88.

632 14. Hardman JG, Ross JJ. Modelling: a core technique in anaesthesia and critical care research. 633 British Journal of Anaesthesia. 2006;97(5):589-92.

634 15. Holford N, Ma SC, Ploeger BA. Clinical trial simulation: a review. Clinical Pharmacology 635 and Therapeutics. 2010;88(2):166-82. 
636 16. Kapellos GE, Alexiou TS, Payatakes AC. Theoretical modeling of fluid flow in cellular

637 biological media: an overview. Mathematical Biosciences. 2010;225(2):83-93.

638 17. Seethala RR, Esposito EC, Abella BS. Approaches to improving cardiac arrest resuscitation 639 performance. Current Opinion in Critical Care. 2010;16(3):196-202.

640 18. McCahon RA, Columb MO, Mahajan RP, Hardman JG. Validation and application of a high641 fidelity, computational model of acute respiratory distress syndrome to the examination of the indices 642 of oxygenation at constant lung-state. British Journal of Anaesthesia. 2008;101(3):358-65.

643 19. Mintun MA, Lundstrom BN, Snyder AZ, Vlassenko AG, Shulman GL, Raichle ME. Blood 644 flow and oxygen delivery to human brain during functional activity: Theoretical modeling and 645 experimental data. Proceedings of the National Academy of Sciences of the United States of 646 America. 2001;98(12):6859-64.

647 20. Noble D. Modeling the heart--from genes to cells to the whole organ. Science. $648 \quad 2002 ; 295(5560): 1678-82$.

649 21. Moppett IK, Hardman JG. Development and Validation of an Integrated Computational 650 Model of Cerebral Blood Flow and Oxygenation. Anesthesia \& Analgesia. 2007;105(4):1094-103.

651 22. Murkin JM, Arango M. Near-infrared spectroscopy as an index of brain and tissue 652 oxygenation. British Journal of Anaesthesia. 2009;103(Supplement 1):i3-i13.

653 23. Hermans MP, Brotons C, Elisaf M, Michel G, Muls E, Nobels F. Optimal type 2 diabetes 654 mellitus management: the randomised controlled OPTIMISE benchmarking study: baseline results 655 from six European countries. European Journal of Preventive Cardiology. 2013;20(6):1095-105.

656 24. Saynor ZL, Barker AR, Oades PJ, Williams CA. A protocol to determine valid VO2max in 657 young cystic fibrosis patients. Journal of Science and Medicine in Sport. 2013;16(6):539-44.

658 25. Saynor ZL, Barker AR, Oades PJ, Williams CA. Reproducibility of maximal 659 cardiopulmonary exercise testing for young cystic fibrosis patients. Journal of Cystic Fibrosis. $660 \quad 2013 ; 12(6): 644-50$.

661 26. Hulzebos HJ, Werkman MS, van Brussel M, Takken T. Towards an individualized protocol 662 for workload increments in cardiopulmonary exercise testing in children and adolescents with cystic 663 fibrosis. Journal of Cystic Fibrosis. 2012;11(6):550-4. 
664 27. Barker AR, Williams CA, Jones AM, Armstrong N. Establishing maximal oxygen uptake in 665 young people during a ramp cycle test to exhaustion. British Journal of Sports Medicine. $6662011 ; 45(6): 498-503$.

667 28. Sacks J, Welch WJ, Mitchell TJ, Wynn HP. Design and analysis of computer experiments. 668 Statistical Science. 1989:409-23.

669 29. Kleijnen JP. Kriging metamodeling in simulation: A review. European Journal of Operational $670 \quad$ Research. 2009;192(3):707-16.

671 30. Jin R, Chen W, Simpson TW. Comparative studies of metamodelling techniques under 672 multiple modelling criteria. Structural and Multidisciplinary Optimization. 2001;23(1):1-13.

673 31. Forrester AI, Keane AJ. Recent advances in surrogate-based optimization. Progress in 674 Aerospace Sciences. 2009;45(1):50-79.

675 32. Chen VC, Tsui K-L, Barton RR, Meckesheimer M. A review on design, modeling and 676 applications of computer experiments. IIE Transactions. 2006;38(4):273-91.

677 33. Schwaighofer A, Grigoras M, Tresp V, Hoffmann C, editors. GPPS: A Gaussian process 678 positioning system for cellular networks. Advances in Neural Information Processing Systems; 2004.

679 34. Bailer-Jones CAL, Sabin TJ, MacKay DJC, Withers PJ, editors. Prediction of deformed and 680 annealed microstructures using Bayesian neural networks and Gaussian processes. Proceedings of the Australasia Pacific Forum on Intelligent Processing and Manufacturing of Materials; 1997.

682 35. Kalaitzis AA, Lawrence ND. A simple approach to ranking differentially expressed gene 683 expression time courses through Gaussian process regression. BMC Bioinformatics. 2011;12(1):180.

684 36. Lawrence ND, Sanguinetti G, Rattray M. Modelling transcriptional regulation using Gaussian 685 processes. Advances in Neural Information Processing Systems. 2007;19:785.

686 37. Swain PS, Stevenson K, Leary A, Montano-Gutierrez LF, Clark IB, Vogel J, et al. Inferring 687 time derivatives including cell growth rates using Gaussian processes. Nature Communications. $6882016 ; 7$.

689 38. Taylor-Robinson D, Whitehead M, Diderichsen F, Olesen HV, Pressler T, Smyth RL, et al. 690 Understanding the natural progression in\% FEV1 decline in patients with cystic fibrosis: a 691 longitudinal study. Thorax. 2012;67(10):860-6. 
692 39. Vilozni D, Bentur L, Efrati O, Minuskin T, Barak A, Szeinberg A, et al. Spirometry in early 693 childhood in cystic fibrosis patients. Chest. 2007;131(2):356-61.

694 40. Kerem E, Reisman J, Corey M, Canny GJ, Levison H. Prediction of mortality in patients with 695 cystic fibrosis. New England Journal of Medicine. 1992;326(18):1187-91.

696 41. Beaver WL, Wasserman K, Whipp BJ. A new method for detecting anaerobic threshold by 697 gas exchange. Journal of Applied Physiology. 1986;60(6):2020-7.

698 42. Rasmussen C, Williams C. Gaussian processes for machine learning (Adaptive computation 699 and machine learning series) Cambridge, MA: The MIT Press; 2005.

700 43. Thin AG, Linnane SJ, McKone EF, Freaney R, FitzGerald MX, Gallagher CG, et al. Use of 701 the gas exchange threshold to noninvasively determine the lactate threshold in patients with cystic 702 fibrosis. Chest. 2002;121(6):1761-70.

703 44. Thin AG, Dodd JD, Gallagher CG, Fitzgerald MX, McLoughlin P. Effect of respiratory rate 704 on airway deadspace ventilation during exercise in cystic fibrosis. Respiratory Medicine. 705 2004;98(11):1063-70.

706 45. Jones DR, Schonlau M, Welch WJ. Efficient global optimization of expensive black-box 707 functions. Journal of Global Optimization. 1998;13(4):455-92.

708 46. Cooper PJ, Robertson CF, Hudson IL, Phelan PD. Variability of pulmonary function tests in 709 cystic fibrosis. Pediatric Pulmonology. 1990;8(1):16-22.

710 47. Balfour-Lynn IM. Personalised medicine in cystic fibrosis is unaffordable. Paediatric 711 Respiratory Reviews. 2014;15 Suppl 1:2-5.

712 48. Titsias MK, editor Variational Learning of Inducing Variables in Sparse Gaussian Processes. 713 AISTATS; 2009.

714 49. Mazzoleni MJ, Battaglini CL, Martin KJ, Coffman EM, Mann BP. Modeling and predicting 715 heart rate dynamics across a broad range of transient exercise intensities during cycling. Sports 716 Engineering. 2016;19(2):117-27.

717 50. Stirling JR, Zakynthinaki M, Refoyo I, Sampedro J. A Model of Heart Rate Kinetics in 718 Response to Exercise. Journal of Nonlinear Mathematical Physics. 2008;15(sup3):426--36.

719 51. Ursino M. Interaction between carotid baroregulation and the pulsating heart: a mathematical 720 model. American Journal of Physiology Heart and Circulatory Physiology. 1998;275(5):H1733-H47. 
721 52. Zakynthinaki MS. Modelling heart rate kinetics. PLoS ONE. 2015;10(4):1--26.

722 53. Gutierrez G. A mathematical model of tissue-blood carbon dioxide exchange during hypoxia.

723 American Journal of Respiratory and Critical Care Medicine. 2004;169(4):525-33.

724 54. Bates JHT. Lung mechanics: an inverse modeling approach. Cambridge: Cambridge 725 University Press; 2009.

726 55. Reynolds A, Ermentrout GB, Clermont G. A mathematical model of pulmonary gas exchange 727 under inflammatory stress. Journal of Theoretical Biology. 2010;264(2):161-73.

728 56. Crooke P, Hotchkiss J, Marini J. Linear and nonlinear mathematical models for noninvasive 729 ventilation. Mathematical and Computer Modelling. 2002;35(11-12):1297-313.

730 57. Kek K, Miyakawa T, Yoneyama S, Kudo N, Yamamoto K, editors. Simulation of exercise731 dependent difference in metabolism with a mathematical model for analyses of measurements using 732 near-infrared spectroscopy. Engineering in Medicine and Biology Society, 2006 EMBS'06 28th 733 Annual International Conference of the IEEE; 2006: IEEE.

734 58. Krauss M, Schaller S, Borchers S, Findeisen R, Lippert J, Kuepfer L. Integrating cellular 735 metabolism into a multiscale whole-body model. PLoS Computational Biology. $736 \quad 2012 ; 8(10):$ e1002750.

737 59. Li Y, Lai N, Kirwan JP, Saidel GM. Computational model of cellular metabolic dynamics in 738 skeletal muscle fibers during moderate intensity exercise. Cellular and Molecular Bioengineering. $7392012 ; 5(1): 92-112$.

740 60. Lai N, Camesasca M, Saidel GM, Dash RK, Cabrera ME. Linking pulmonary oxygen uptake, 741 muscle oxygen utilization and cellular metabolism during exercise. Annals of Biomedical 742 Engineering. 2007;35(6):956-69.

743 61. Cloutier M, Wellstead P. The control systems structures of energy metabolism. Journal of The 744 Royal Society Interface. 2009:rsif20090371.

745 62. Dash RK, Dibella Ja, Cabrera ME. A computational model of skeletal muscle metabolism 746 linking cellular adaptations induced by altered loading states to metabolic responses during exercise. 747 Biomedical Engineering Online. 2007;6:14.

748 63. Kim J, Saidel GM, Cabrera ME. Multi-scale computational model of fuel homeostasis during 749 exercise: effect of hormonal control. Annals of Biomedical Engineering. 2007;35(1):69-90. 
750 64. Fresiello L, Meyns B, Di Molfetta A, Ferrari G. A Model of the Cardiorespiratory Response

751 to Aerobic Exercise in Healthy and Heart Failure Conditions. Frontiers in Physiology. 2016;7.

752 65. Fresiello L, Ferrari G, Di Molfetta A, Zieliński K, Tzallas A, Jacobs S, et al. A cardiovascular 753 simulator tailored for training and clinical uses. Journal of Biomedical Informatics. 2015;57:100-12.

754 66. Timischl S. A Global Model for the Cardiovascular and Respiratory System: University of 755 Graz; 1998.

756 67. D'Angelo C. Multiscale modelling of metabolism and transport phenomena in living tissues:

757 École Polytechnique Fédérale de Lausanne; 2007.

758 68. Hardman J, Bedforth N, Ahmed A, Mahajan R, Aitkenhead A. A physiology simulator: 759 validation of its respiratory components and its ability to predict the patient's response to changes in 760 mechanical ventilation. British Journal of Anaesthesia. 1998;81(3):327-32.

761 69. Lai N, Saidel GM, Grassi B, Gladden LB, Cabrera ME. Model of oxygen transport and 762 metabolism predicts effect of hyperoxia on canine muscle oxygen uptake dynamics. Journal of 763 Applied Physiology. 2007;103(4):1366-78.

764 70. Benson AP, Grassi B, Rossiter HB. A validated model of oxygen uptake and circulatory 765 dynamic interactions at exercise onset in humans. Journal of Applied Physiology. 2013;115(5):74376655.

767 71. Bell C, Paterson DH, Kowalchuk JM, Padilla J, Cunningham DA. A Comparison of 768 Modelling Techniques used to Characterise Oxygen Uptake Kinetics During the on-Transient of 769 Exercise. Experimental Physiology. 2001;86(5):667-76.

770 72. Elstad M, Toska K, Walløe L. Model simulations of cardiovascular changes at the onset of 771 moderate exercise in humans. The Journal of Physiology. 2002;543(2):719-28.

772 73. $\mathrm{Ng} \mathrm{LJ}$, Sih BL, Stuhmiller JH. An integrated exercise response and muscle fatigue model for 773 performance decrement estimates of workloads in oxygen-limiting environments. European Journal 774 of Applied Physiology. 2012;112(4):1229-49.

775 74. Walter G, Vandenborne K, Elliott M, Leigh JS. In vivo ATP synthesis rates in single human 776 muscles during high intensity exercise. The Journal of Physiology. 1999;519(3):901-10. 
75. Eriksson A, Holmberg H-C, Westerblad H. A numerical model for fatigue effects in wholebody human exercise. Mathematical and Computer Modelling of Dynamical Systems. 2016;22(1):2138.

76. Liu JZ, Brown RW, Yue GH. A dynamical model of muscle activation, fatigue, and recovery.

Biophysical Journal. 2002;82(5):2344-59.

782 77. Dash RK, Li Y, Kim J, Saidel GM, Cabrera ME. Modeling cellular metabolism and 783 energetics in skeletal muscle: large-scale parameter estimation and sensitivity analysis. IEEE 784 Transactions on Biomedical Engineering. 2008;55(4):1298-318.

785 78. Moxnes JF, Sandbakk $\varnothing$. The kinetics of lactate production and removal during whole-body 786 exercise. Theoretical Biology and Medical Modelling. 2012;9(1):7.

787 79. Péronnet F, Aguilaniu B. Lactic acid buffering, nonmetabolic CO 2 and exercise 788 hyperventilation: a critical reappraisal. Respiratory Physiology \& Neurobiology. 2006;150(1):4-18.

789 80. Batzel JJ, Kappel F, Timischl-Teschl S. A cardiovascular-respiratory control system model 790 including state delay with application to congestive heart failure in humans. Journal of Mathematical 791 Biology. 2005;50(3):293-335.

792 81. Hebestreit H, Arets HG, Aurora P, Boas S, Cerny F, Hulzebos EH, et al. Statement on 793 Exercise Testing in Cystic Fibrosis. Respiration. 2015;90(4):332-51.

794 82. Weir E, Burns PD, Devenny A, Young D, Paton JY. Cardiopulmonary exercise testing in 795 children with cystic fibrosis: one centre's experience. Archives of disease in childhood. $796 \quad 2017 ; 102(5): 440-4$.

797 83. Liou TG, Adler FR, Fitzsimmons SC, Cahill BC, Hibbs JR, Marshall BC. Predictive 5-Year

798 Survivorship Model of Cystic Fibrosis. American Journal of Epidemiology. 2001;153(4):345-52.

799 84. Aaron SD, Stephenson AL, Cameron DW, Whitmore GA. A statistical model to predict one800 year risk of death in patients with cystic fibrosis. Journal of Clinical Epidemiology. $801 \quad 2015 ; 68(11): 1336-45$.

802 85. Middleton PG, Matson AG, Robinson PD, Jane Holmes-Walker D, Katz T, Hameed S. Cystic 803 Fibrosis Related Diabetes: Potential pitfalls in the transition from paediatric to adult care. Paediatric 804 Respiratory Reviews. 2014;15(3):281-4. 
805 86. Hayes D, Jr., Tobias JD, Mansour HM, Kirkby S, McCoy KS, Daniels CJ, et al. Pulmonary

806 hypertension in cystic fibrosis with advanced lung disease. American Journal of Respiratory and

807 Critical Care Medicine. 2014;190(8):898-905.

808

809 


\section{$810 \quad$ Figure Titles}

811 Figure 1: (a) The work rate for each participant is increased at a rate dependent on their past

812 test performance. (b) Participant age is correlated with test performance for young

813 participants, but not for older ones.

814

815 Figure 2: Ratio of oxygen utilisation and total breathing throughout the test. Markers indicate 816 the volitional exhaustion times for each participant.

Figure 3: (a) Correlation of $\mathrm{FEV}_{1}$ with the maximal tidal volume achieved throughout the test. (b) Correlation between FEV $F_{1}$ and FVC is high. Note that, although FVC and FEV 1 are good predictors of poor test performance, they are unable to distinguish better performing participants.

Figure 4: (a) Total ventilation plotted against oxygen utilisation. We observe that breathing pattern is strongly correlated with test performance. (b) Exponential curves are fitted through the raw data, further highlighting this dependence.

Figure 5: Slope of the fitted curves (log $\dot{V} E$ against $\left.\dot{V} O_{2}\right)$ from Fig. 4(b) plotted against the total energy transfer during the test. We find a relatively poor characterisation of the variance between performances. (b) By instead plotting the oxygen consumption at a fixed rate of breathing, we better capture differences in performance.

Figure 6: (a) Breathing patterns subdivided into the breathing rate and tidal volume. These data appear uninformative for predicting test performance. (b) With the additional inclusion of the oxygen consumption at a fixed rate of breathing, we find that these variables now almost perfectly capture variation in participant performance.

Figure 7: First of the principal components obtained via PCA accounts for over 90\% of the variation in test performance. (b) Similar levels of variance are accounted for by taking only the normal component of the first principal component in the breathing frequency direction. 
841 Figure 8: (a) Quantifying the relationship between the anaerobic threshold and overall test

842 performance. Anaerobic thresholds were calculated using an automated procedure based on

843 previous methods (41) (b) $\dot{\mathbf{V}} \mathbf{O}_{2 \max }$ is the best single predictor of overall test performance.

845 Figure 9: GP is a probability distribution on function spaces. The thick solid red line is the true

846 function $f$ and the thick dashed black line is the GP mean. (a) Thin grey lines show sample

847 paths of a prior GP whose mean is zero. (b) Blue bullets indicate five data points sampled from

$848 f$. The GP mean and covariance are updated using these sample points. The thin grey dashed

849 lines show $m(\mathbf{x}) \pm 2 s(\mathbf{x})$.

851 Figure 10: (a) Predictions (asterisks) vs. exact values (bullets). Red bars show the 95\% 852 confidence intervals, $\boldsymbol{m}_{\mathrm{i}}\left(\mathrm{x}_{\mathrm{i}}\right) \pm 1.96 \boldsymbol{s}_{i}\left(\mathrm{x}_{\mathrm{i}}\right)$ around the predicted value, where $\boldsymbol{m}_{\mathrm{i}}$ and $s_{\mathrm{i}}$ are GP 853 prediction mean and standard deviation based on all but the $i$ 'th data point. (b) Relative 854 prediction error expressed as a percentage of the true value $f\left(\mathbf{x}_{\mathbf{i}}\right)$.

856 Figure 11: Schematic of the variables and processes in the proposed ODE-based mathematical 857 model. Adapted from Timischl (1998) (66) and Batzel et al. (2005) (80). 